\title{
Médiévales
}

Langues, Textes, Histoire

62 | printemps 2012

Hagiographie et réforme dans l'Occident latin

\section{Réformer la vie monastique ou réformer l'Empire? La Vie d'Eigil de Fulda par Brun Candidus (vers 840)}

Reforming Monastic Life or Reforming Empire? The Life of Eigil of Fulda by Brun Candidus (c. 840)

\section{Klaus Krönert}

\section{OpenEdition}

\section{Journals}

Édition électronique

URL : https://journals.openedition.org/medievales/6645

DOI : 10.4000/medievales.6645

ISSN : 1777-5892

Éditeur

Presses universitaires de Vincennes

Édition imprimée

Date de publication : 2 juin 2012

Pagination : 49-65

ISBN : 978-2-84292-346-4

ISSN : 0751-2708

\section{Référence électronique}

Klaus Krönert, «Réformer la vie monastique ou réformer l'Empire? La Vie d'Eigil de Fulda par Brun

Candidus (vers 840) ", Médiévales [En ligne], 62 I printemps 2012, mis en ligne le 28 juin 2012, consulté le 24 avril 2022. URL : http://journals.openedition.org/medievales/6645 ; DOI : https://doi.org/

10.4000/medievales.6645 
Médiévales 62, printemps 2012, p. 49-66

\author{
Klaus KRÖNERT
}

\title{
RÉFORMER LA VIE MONASTIQUE OU RÉFORMER L'EMPIRE? LA VIE D'EIGIL DE FULDA PAR BRUN CANDIDUS (VERS 840)
}

Si le thème de la «réforme» est sous-jacent dans presque toutes les Vies de saint - un saint représente un idéal et il est donc un miroir de l'imperfection du lecteur -, certaines Vies sont plus explicitement rattachées à un contexte de réforme particulier, comme la Vie d'Eigil (BHL 2440-2441) ${ }^{1}$. Écrite à Fulda vers 840 par Brun Candidus, elle est consacrée au quatrième abbé du grand monastère de la Hesse fondé en 744 à la demande de Boniface par l'un de ses disciples, Sturmi (779), et devenu dans les premières décennies du IX ${ }^{\mathrm{e}}$ siècle un centre très actif de la Renaissance carolingienne où séjournèrent de grands savants comme Walahfrid Strabon, Gottschalk, Éginhard, Loup de Ferrières et, bien sûr, son célèbre abbé Raban Maur (822-842)². Cette ascension fulgurante n'alla

1. Cette étude fait partie d'un ensemble de travaux sur la production hagiographique en Germanie à l'époque de Louis le Pieux, travaux que j'ai engagés dans le cadre du projet «Hludowicus » (ANR-DFG) dirigé par Stefan Esders (FU Berlin) et Philippe Depreux (Université de Limoges). Je tiens à remercier tous les membres de ce groupe pour leurs conseils et leurs remarques critiques, ainsi que Damien Kempf et les participants de la table ronde lilloise où j'ai présenté une première version de ce travail.

2. La bibliographie relative à l'histoire du monastère de Fulda au haut Moyen Âge est abondante. Mentionnons seulement les études fondamentales pour notre sujet: P. ENGELBERT, Die Vita Sturmi des Eigil von Fulda. Literarkritisch-historische Untersuchung und Edition, Marburg, 1968 ; K. HeinemeYer, «Die Gründung des Klosters Fulda im Rahmen der bonifatianischen Kirchenorganisation », Hessisches Jahrbuch für Landesgeschichte, 30 (1980), p. 1-45 ; G. SCHRIMPF éd., Kloster Fulda in der Welt der Karolinger und Ottonen. Kultur - Politik - Wirtschaft, Fulda, 1996; K. Schmid éd., Die Klostergemeinschaft von Fulda im frühen Mittelalter, 3 vol., Munich, 1978 ; J. E. RAAIJMAKERS, Sacred Time, Sacred Space. History and Identity at the monastery of Fulda (744-856), thèse soutenue à l'université d'Amsterdam en 2003, en cours de publication aux Presses de l'Université de Cambridge. 
cependant pas sans difficultés, comme l'atteste la Vie d'Eigil. Le texte relate de manière détaillée les problèmes que connut la communauté lorsque s'imposa la réforme monastique de 816/817. Toutefois, les relations entre réforme et écriture hagiographique s'avèrent complexes. Une analyse de la Vie montre que son auteur était beaucoup moins préoccupé par les faits de 816/817 (qu'il décrit pourtant in extenso) que par les nouvelles crises de la fin des années 830 dans lesquelles se trouvaient plongés le monastère et l'Empire. Il apparait que Brun Candidus, en composant la Vie d'Eigil, entendait donner aux acteurs de son époque une sorte de guide, rappelant comment un abbé avait résolu une crise grave une bonne vingtaine d'années plus tôt, pour mieux indiquer à ses contemporains comment sortir de celle qu'ils traversaient à leur tour.

\section{La Vita Eigilis de Brun Candidus}

La Vita Eigilis est aujourd'hui accessible dans l'édition procurée par Georg Waitz et Ernst Dümmler dans la collection des Monumenta Germaniae Historica. Elle a été rééditée récemment par Gereon Becht-Jördens ${ }^{3}$. Il en existe deux traductions partielles, dues à Georg Grandauer et à Dominikus Heller ${ }^{4}$. Malgré le caractère exceptionnel de l'œuvre, la Vie n'a que très peu retenu l'attention des chercheurs jusqu'à une époque récente. L'étude de Walter Berschin ${ }^{5}$, l'analyse prosopographique de Pius Engelbert ${ }^{6}$, la nouvelle édition commentée de Gereon Becht-Jördens, ainsi que plusieurs articles de cet érudit, lui ont cependant rendu la place qu'elle méritait dans l'histoire des textes ${ }^{7}$. En 2003, Janneke Raaijmakers a consacré une thèse de doctorat à l'histoire de Fulda au début du IX ${ }^{e}$ siècle où il est amplement question de la Vie d'Eigil ${ }^{8}$.

3. Brun Candidus, Vita Eigilis pros. et metr., éd. G. Waitz, MGH, SS, 15, Hanovre, 1887, p. 222-233; éd. E. DümmLer MGH, Poetae latini aevi Carolini, 2, Hanovre, 1884, p. 94-117; nouvelle édition par G. BECHT-JÖRDENs éd., Vita Aegil abbatis Fuldensis a Candido ad modestum edita prosa et versibus. Ein opus geminum des Ix. Jahrhunderts. Einleitung und kritische Edition, Marburg, 1994; à propos des manuscrits, voir ibid. et J. E. RaAiJMakers, Sacred Time, Sacred Space..., p. 143 et $s q$.

4. G. Grandauer, Leben des Abtes Eigil von Fulda, Leipzig, 1890 ; D. Heller éd., Die ältesten Geschichtsschreiber des Klosters Fulda, Fulda, 1952.

5. W. Berschin, «Biographie im karolingischen Fulda», dans Kloster Fulda..., p. 315-25; ID., Biographie und Epochenstil im lateinischen Mittelalter, t. 3, Karolingische Biographie, 750920 n. Chr., Stuttgart, 1991, p. 254-258.

6. P. Engelbert, Die Vita Sturmi..., p. 5-15.

7. G. BeCht-JöRdens, Die Vita Aegil abbatis Fuldensis des Brun Candidus. Ein opus geminum aus dem Zeitalter der anianischen Reform in biblisch-figuralem Hintergrundstil, Francfort-sur-leMain, 1992; G. BECHT-JöRDENs, «Die Vita Aegil des Brun Candidus als Quelle zu Fragen aus der Geschichte Fuldas im Zeitalter der anianischen Reform », Hessisches Jahrbuch für Landesgeschichte, 42 (1992), p. 19-48.

8. Voir supra n. 2. 
Les informations que nous possédons au sujet de son auteur, Brun, appelé aussi Brun Candidus, sont plutôt rares : nous savons seulement qu'il a connu, en tant que moine de Fulda, trois abbés, Ratgar (802-817), Eigil (818-822) et Raban Maur (822-842). Brun exerça la charge de scribe - un manuscrit de la Regula Benedicti copié par lui nous est parvenu -, et il a reçu une formation artistique qui lui permit de peindre la nouvelle abside occidentale de la basilique SaintSauveur de Fulda dans laquelle reposait, depuis 819, la tombe de Boniface certainement grâce à Éginhard, comme l'indiquent les Gesta abbatum (qui sont en fait une liste annotée d'abbés du IX ${ }^{\mathrm{e}}$ ou X $\mathrm{X}^{\mathrm{e}}$ siècle). Qu'il soit devenu le successeur de Raban Maur à la tête de l'école monastique est possible, mais non prouvé. Brun est mort, semble-t-il, en 845 : c'est à cette date que l'on trouve son nom dans une notice nécrologique du monastère ${ }^{9}$.

Composée vers 840, la Vie d'Eigil fait partie d'une série de Vies d'abbés ${ }^{10}$. Ces Vitae abbatum de Fulda forment une collection de biographies pour laquelle il n'y avait pas de modèle en Occident à cette époque. Eigil a ainsi écrit, vers 818-820, une Vita Sturmi (BHL 7924) ${ }^{11}$; Brun Candidus, peu de temps après, une Vita Baugulfi ${ }^{12}$; un auteur anonyme, sans doute à la même époque, une Vita Ratgarii ${ }^{13}$; Brun, vers 840, la Vita Eigilis, dont il est question ici ; et Rodolphe, vers 842/847, la Vita Rabani, texte qui est aussi connu sous le titre de Miracula sanctorum in Fuldenses ecclesias translatorum (BHL 7044) ${ }^{14}$. Ces Vies d'abbés

9. À propos de la biographie de Brun Candidus, voir F. BRUNHÖLZL, Histoire de la littérature latine du Moyen Âge, t. 1-2, L'époque carolingienne, Turnhout, 1991, p. 99 et sq.; W. BERsChin, Biographie und Epochenstil..., p. 241 et sq.; G. Becht-Jördens éd., Vita Aegil..., p. XII; J. E. RaAijmakers, Sacred Time, Sacred Space..., p. 123-125 et 141 et sq.

10. Cf. W. Berschin, Biographie und Epochenstil..., p. 240 ; J. E. RaAijmakers, Sacred Time, Sacred Space..., p. 124. Brun mentionne dans la Vita, lib. II, cap. 7, que le frère Recceno venait de mourir, or les annales necrologi précisent que Recceno est mort en 839. De plus, l'auteur appelle Raban «abbé», ce qui prouve que le texte a été écrit avant 842. L'ensemble de l'œuvre suggère enfin que Louis le Pieux, mort le 20 juin 840, était encore en vie.

11. Pour la majorité des chercheurs, la Vie de Sturmi aurait été écrite du vivant de Charlemagne, donc avant 814, et peut-être même avant 803: cf. P. Engelbert, Die Vita Sturmi..., p. 18-20; et S. Patzold, «Raban, Gottschalk und der Traktat "De oblatione puerorum"», dans P. Depreux, S. LebecQ, M. J.-L. Perrin et O. Szerwiniack éd., Raban Maur et son temps, Turnhout, 2010, p. 105118, spéc. p. 106 et suiv.; J. E. RaAiJmaKers, Sacred Time, Sacred Space..., p. 68-82, a cependant montré, de manière très convaincante, que la Vie de Sturmi avait été écrite dans les années 818-820 avec l'objectif de clore la querelle ouverte par l'abbatiat de Ratgar et la construction de la nouvelle église abbatiale, en établissant une tradition dans laquelle tous les moines de Fulda, jusque-là divisés, pouvaient se retrouver.

12. J. E. RAAijmakers, Sacred Time, Sacred Space..., p. 137; à propos de l'ancienne datation de cette Vie, vers 802, voir W. BERSCHIN, Biographie und Epochenstil..., p. 240.

13. Cf. J. E. RaAijmakers, Sacred Time, Sacred Space..., p. 137; à propos de l'ancienne datation de cette Vie, après 817, voir W. BERschin, Biographie und Epochenstil..., p. 240.

14. W. BeRsChin, Biographie und Epochenstil..., p. 240. 
ne sont pas conçues comme une série de notices, comme, par exemple, les Gesta des abbés de Fontenelle (Saint-Wandrille), mais comme des Vies indépendantes. Leur continuité reste tout de même étonnante. Hélas, la plus grande partie de la bibliothèque de Fulda a disparu au XVII ${ }^{\mathrm{e}}$ siècle, lors de la Guerre de Trente ans, et il nous manque ainsi les deux Vies composées entre la Vita Sturmi et la Vita Eigilis ${ }^{15}$.

La Vie d'Eigil a encore une autre particularité: Brun ne compte pas explicitement Eigil au nombre des saints ${ }^{16}$. Nous n'avons cependant aucune raison de douter que l'auteur a bel et bien écrit un texte hagiographique, considérant son héros comme un «ami de Dieu», pour reprendre une expression de Guy Philippart. Le savant belge propose cette expression pour éviter les questions épineuses et finalement peu fructueuses sur les «saintes personnes» dont le culte n'avait pas pris, dont la vénération a été uniquement populaire et dont la canonisation a été retardée ou avortée ${ }^{17}$. On sait que le haut Moyen Âge ne connaissait pas encore de catégories aussi strictes que les époques plus tardives. Assurément, la Vie d'Eigil est bien plus qu'une simple biographie, comme le montrera notre analyse.

Brun a conçu un opus geminum illustré, c'est-à-dire une Vie divisée en deux parties, dont la première relate en prose les hauts faits du héros (BHL 2440), et la deuxième en vers (BHL 2441). Cette dernière, appelée «second livre», était illustrée par une série de miniatures, ce qui était alors une nouveauté. Les images nous sont connues grâce à l'editio princeps de Christoph Brower datant de 1616, qui a reproduit trois miniatures d'un codex de Fulda aujourd'hui perdu ${ }^{18}$.

L'hagiographe a dédié la Vie d'Eigil à l'un de ses confrères, Modestus, qui est aussi présenté par Christoph Brower comme l'illustrateur du deuxième livre. Une dédicace à un simple confrère n'est pas chose courante. On aurait plutôt attendu que l'auteur choisisse l'abbé de la communauté, Raban Maur. Le sens profond d'un tel hommage se dévoile en étudiant l'image de dédicace: normalement l'auteur, de petite taille, soumet son travail à son commanditaire, représenté dans de plus grandes proportions. Ici, l'auteur montre son livre à un confrère et les deux personnages sont représentés à taille égale, discutant du contenu du texte. Il fait peu de doute que Modestus représente la communauté de Fulda. L'œuvre avait assurément une visée didactique : elle était d'abord

15. Ibid., p. 241.

16. Ibid., p. 253.

17. G. PhilipParT, «L'hagiographie, histoire sainte des "amis de Dieu”», dans ID. éd., Hagiographies, t. 4, Turnhout, 2007, p. 13-40.

18. W. Berschin, Biographie und Epochenstil...,p. 250-258; J. E. RAAIJMAKERs, Sacred Time, Sacred Space ..., p. 123-125; G. BECHT-JöRDENS éd., Vita Aegil..., p. XI-XXVIII ; pour l'editio princeps de la Vie, voir C. Brower, Antiquitatum Fuldensium libri IV, Anvers, 1612 (les trois enluminures sont reproduites aux p. 90 et 170). 
destinée aux moines ${ }^{19}$. Il n'empêche que Brun précise bien que le commanditaire ou plutôt l'instigateur du texte était Raban Maur: dans le prologue, il explique que, ne trouvant pas suffisamment de frères érudits dans la communauté pour discuter de problèmes théologiques, Raban lui avait suggéré de poursuivre seul sa formation par la lecture et l'écriture d'une «œuvre utile», comme l'illustre abbé l'avait fait lui-même avec le traité De laudibus sanctae crucis. C'est ce qui a décidé Brun à entreprendre la rédaction de la Vie d'Eigil en prose et en vers ${ }^{20}$.

Le genre de l'opus geminum remonte au v siècle, avec le Carmen pascale (en vers) et l'Opus pascale (en prose) de Sedulius reprenant les hauts faits de la vie du Christ d'après les Évangiles. Aux viI ${ }^{\mathrm{e}}$-VIII ${ }^{\mathrm{e}}$ siècles, l'Anglo-saxon Aldhelm, auteur d'un autre opus geminum, le De virginitate, explique qu' il a mis en prose et en vers le contenu de son œuvre afin d'exploiter toutes les possibilités linguistiques pour reproduire à l'aide des mots la création qui, par sa complexité, est en elle-même un éloge de Dieu, mais réalisée d'une «voix différente » (diversa voce). Bède - avec ses Vies de Cuthbert - et Alcuin - avec ses Vies de Willibrord - ont eu des motivations similaires ${ }^{21}$. Brun Candidus eut, lui aussi, des raisons particulières de relater ainsi les hauts faits d'Eigil: la Vie en prose contient davantage de passages «politiques» et «événementiels», tandis que la Vie en vers met l'accent sur des aspects cultuels, culturels et ecclésiologiques ${ }^{22}$.

\section{Eigil et le retour de l'unité}

Brun débute la Vie en prose par la présentation de son héros. D'origine bavaroise, Eigil fut offert au monastère de Fulda et, après une scolarité exemplaire, devint moine et prêtre ${ }^{23}$. L'auteur détaille la succession des abbés du monastère et aborde les problèmes internes de la communauté apparus lors de l'abbatiat de Ratgar $^{24}$.

19. Brun Candidus, Vita Aegil abbatis Fuldensis, lib. II, prologue, (éd. G. Becht-Jördens, p. 31); cf. également W. Berschin, Biographie une Epochenstil..., p. 254-258; G. BeCHT-JöRdens, Die Vita Aegil abbatis..., p. 23-25 ; J. E. RaAjumakers, Sacred Time, Sacred Space..., p. 123-125 et 143-144.

20. Brun CAndidus, Vita Aegil abbatis Fuldensis, lib. I, prologue (éd. G. BeChT-Jördens, p. 3-4).

21. À propos de l'opus geminum, voir P. KLOPSCH, «Prosa und Vers in der mittellateinischen Literatur», Mittellateinisches Jahrbuch, 3 (1966), p. 9-24; P. Goldman, «The Anglo-Latin Opus Geminatum: from Aldhelm to Alcuin », Medium Aevum, 50-2 (1981), p. 215-229; G. BECHT-JöRDENS, Die Vita Aegil abbatis..., p. 26 et $s q$.

22. J. E. RaAjmakers, Sacred Time, Sacred Space..., p. 123-139 et 144-150.

23. Brun Candidus, Vita Aegil abbatis Fuldensis, lib. I, cap. 1-2 (éd. G. Becht-Jördens, p. 5).

24. Ibid., lib. I, cap. 3 (éd. G. BeCht-Jördens, p. 5). 
Cette crise a fait couler beaucoup d'encre ${ }^{25}$. Présentons ici rapidement les faits : après des moments difficiles vers 807 à cause d'une épidémie, un premier conflit éclata en 812. Plusieurs moines se présentèrent devant l'empereur avec une sorte de pétition, le supplex libellus. Au cœur du conflit se trouvait la construction d'une nouvelle église abbatiale considérablement plus grande que la précédente, lancée par l'abbé Baugulf qui avait désigné Ratgar comme architecte et maître des travaux. Élu abbé après la mort de Baugulf, Ratgar poursuivit ces travaux qui épuisaient littéralement la communauté. En 816/817, les moines de Fulda envoyèrent une seconde délégation auprès de l'empereur (désormais Louis le Pieux). De manière plus générale, les moines reprochaient à Ratgar son manque de modestie, sa «démesure», en fait son absence de sagesse, en contradiction avec l'idéal bénédictin ${ }^{26}$. Louis le Pieux le destitua alors de sa charge et envoya deux missi - Aaron et Adalfrid - afin d'apporter la paix à la communauté, de corriger les moines dont le comportement laissait à désirer et, enfin, d'organiser l'élection d'un nouvel abbé ${ }^{27}$. Eigil, qui était arrivé à Fulda dans les années 750 , fut alors choisi.

Ces événements sont rapidement évoqués par Brun Candidus au début de sa Vita prosaica. L'auteur accorde d'abord à l'élection d'Eigil une importance particulière: l'hagiographe rapporte longuement, le plus souvent au discours direct, comment les moines cherchèrent un candidat en hésitant entre plusieurs «profils»: entre des origines nobles ou simples, entre un pauvre et un riche, entre un érudit ou un pragmatique ${ }^{28}$. Certains moines décidèrent alors d'interroger les candidats eux-mêmes sur leurs intentions en cas d'élection ${ }^{29}$. Ce passage nous rappelle que des procédures électives n'étaient pas étrangères au Moyen Âge, comme l'a remarqué Walter Berschin ${ }^{30}$. Eigil, le vainqueur de cette élection, est

25. Cf., à propos des crises de Fulda des années 812-817, M. Gaillard, D'une réforme à l'autre (816-934): les communautés religieuses en Lorraine à l'époque carolingienne, Paris, 2006; J. SemmLER, «Studien zum Supplex Libellus und zur anianischen Reform in Fulda», Zeitschrift für Kirchengeschichte, 69 (1958), p. 268-98; G. Becht-Jördens, Die Vita Aegil abbatis...; J. E. RaAijmakers, Sacred Time, Sacred Space...

26. J. E. RaAimakers, Sacred Time, Sacred Space..., p. 65.

27. Ibid., p. 67.

28. BRUn CANDidus, Vita Aegil abbatis Fuldensis, lib. I, cap. 5: «Quiescite, fratres, ab hac alta et generosa electione, quia infirma mundi et contemptibilia elegit Deus. Infirmitas namque in Deo innocentia est, ignobilitas autem humilitas. Nam innocentia sine Deo infirmitas aestimatur, et humilitas non propter Deum ignobilitas iudicatur. Tali etiam, non dico nobilitati, sed ignobilitati si colla subiicimus, quo nos vertimus, si aversus fuerit a nobis, opprimens? Si ei humiliter suggerimus, ut quiescat, fortasse maiori commotus iracundia non quiescit, quia confidentiam habet in propinquis » (éd. G. BeCht-Jördens, p. 6-7).

29. Ibid.: «Si te vel te nobis praeficimus, dic nobis, oramus, in veritate, si velis tunc nobis facere bene.»

30. W. Berschin, Biographie und Epochenstil..., p. 245. 
décrit comme «moralement ferme», «sage», «dur avec les indisciplinés», mais «clément avec les frères aimables afin qu'ils progressent». D'apparence et de caractère «joyeux », il est également qualifié comme un «ami de la paix» et un «homme simple qui n'apprécie guère la ruse $»^{31}$.

Une fois élu, le nouvel abbé fut envoyé au palais impérial d'Aix-laChapelle où Louis le Pieux le confirma, non sans avoir prononcé deux discours d'exhortation qui occupent à nouveau plusieurs pages de la Vie. Ils forment, d'une certaine manière, le cœur de la première partie de l'œuvre. L'un de ces discours est particulièrement intéressant car l'empereur - en réalité Brun et sans doute aussi Raban Maur, l'instigateur de la Vie - y présente son idéal monastique: la communauté doit être une communauté de charité, d'amour et de concorde ${ }^{32}$. Humilité, obéissance et pardon sont des vertus décisives dans la vie monastique; l'orgueil, la querelle et la discorde ne doivent pas trouver leur place dans une abbaye ${ }^{33}$. Si l'humanité ne se confie pas à Dieu, elle est perdue. L'espoir vient, selon Brun, de la grâce que Dieu accorde à celui qui confesse ses péchés et pratique une pénitence sincère ${ }^{34}$. Le dernier tiers du texte est consacré à une visite qu'Eigil rendit à l'archevêque de Mayence, Heistulf, ainsi qu'aux travaux qu'il engagea à Fulda: un nouveau pavement dans l'église, deux cryptes, une nouvelle chapelle octogonale et un nouveau dortoir. De plus, l'auteur rapporte quelques mesures prises par Eigil concernant la vie communautaire, et notamment la lecture au réfectoire de la Vie de Sturmi (qu'il avait écrite), ainsi

31. Brun CAndidus, Vita Aegil abbatis Fuldensis, lib. I, chap. 6: «Erat enim constans et bene cordatus, duri cordis et indisciplinatis durus, mitibus vero et bene morigeratis, ut in melius proficerent, mitis. Eratque hilaris facie, laetus mente, discretus in opere, consentiens in utilitate». Les autres termes employés sont quietis amator et vir simplex (éd. G. BeCHT-JöRdENs, p. 8).

32. Brun Candidus, Vita Aegil abbatis Fuldensis., lib. I, cap. 9: «Nam prima legis in monte Sinai ordinatio haec ad Moysen data est: 'diliges', inquit, 'Dominum Deum tuum ex toto corde tuo et ex tota mente tua et ex tota virtute tua et ex tota anima tua, secundo proximum tuum sicut te ipsum'. Vacat enim dilectio Dei nisi subiungatur et proximi. Plenum ergo praeceptum est veritatis, ut maneat in sensu individua regula caritatis. Cura ergo disciplinae monachorum perfecta dilectio est. Haec vinculum caritatis et pacis exhortantur sine avaritia. Igitur in dilectione sincera modo sufficiens est praesentibus, et qui invicem diligunt, filii regni vocantur perfecte invicem diligentes. Ipsi sunt, qui manducant panem in regno Dei, sine macula vivunt et alienae ruga discordiae» (éd. G. BECHT-JÖRDENS, p. 9-11).

33. Cf. également G. BeCht-Jördens, Die Vita Aegil abbatis..., p. 31 ; J. E. RAAIJMAKERs, Sacred Time, Sacred Space..., p. 146.

34. Brun CAndidus, Vita Aegil abbatis Fuldensis, lib. I, cap. 24 : «Haec namque ideo testimonia Divinae lectionis in hoc libro, frater Modeste, notavi, ut non sit spes sibi quisque, sed ponat in Deo spem suam, et qui se existimat stare, videat, ne cadat, et qui lapsus per superbiam cadat, Divina lectione corresptus studeat cum Dei auxilio resurgere per humilitatem, et ut omnis peccator in ecclesia constitutus cum fide recta per humilem confessionem veramque paenitentiam a Deo veniam speret» (éd. G. Becht-Jördens, p. 19); voir également J. E. RaAjmakers, Sacred Time, Sacred Space..., p. 148 . 
que l'organisation des repas par des églises dépendantes de Fulda. Enfin, nous apprenons qu'Eigil, déjà très affaibli, avait préparé la tombe dans laquelle il fut enterré peu de temps après ${ }^{35}$. Par ses actes, Eigil répondait à l'idéal du bon abbé : être un père qui aime ses fils, qui est humble et sage, et qui maintient le juste équilibre entre le désir du renoncement au monde et les devoirs d'un monastère royal dans le siècle. Tous les moines étaient incités à s'inspirer de son exemple et à corriger leur comportement. À cette fin, l'empereur avait le droit, et même le devoir, d'intervenir dans la communauté monastique ${ }^{36}$.

Il ne fait aucun doute qu'Egil a joué un rôle important pour que le monastère de Fulda retrouve son unité. Ceci est certainement lié principalement à sa façon de diriger la communauté, car Eigil non seulement termina les travaux en cours, mais fit en plus construire de nouveaux bâtiments dont un cloître et une chapelle funéraire ${ }^{37}$. Il était manifestement un homme de consensus, capable de créer une adhésion autour de lui. Janneke Raaijmakers a très clairement montré comment il était arrivé à transformer la nouvelle église abbatiale, symbole de querelle, en véritable ecclesia au sens spirituel du terme ${ }^{38}$. C'est certainement aussi durant cette période que le nouvel abbé écrivit la Vie du fondateur de Fulda, la Vita Sturmi (BHL 7924), dans laquelle il présente son héros comme modèle et montre que la communauté s'appuie sur une double tradition associant les enseignements de Boniface et la Règle de saint Benoît. Eigil incarnait l'abbé sage, modeste et modéré qui arrivait à concilier deux camps dont le premier, plutôt conservateur, représentait un monachisme «à l'ancienne», selon lequel les moines devaient mener une vie ascétique à l'écart du monde. L'autre courant, incarné par Ratgar et ses fidèles, était favorable à la transformation du monastère en un grand centre culturel au service du pouvoir comme l'envisageait la réforme carolingienne. Le principal problème concernait les coutumes à observer, c'està-dire touchait à l'organisation quotidienne de la vie monastique. En revanche, et contrairement à ce que l'on a longtemps cru, la revalorisation de la Règle de saint Benoît depuis les conciles d'Aix de 816/817, n'a pas contribué à diviser la communauté de Fulda: d'une part, l'instigateur anglo-saxon de la fondation de Fulda, Boniface, l'avait reprise en grande partie dans ses enseignements, et d'autre part, Benoît d'Aniane ne voulait pas une rupture brutale en 816/817. Son Codex regularum et sa Concordia regularum contiennent des passages de toutes les règles monastiques, et le réformateur souhaitait que les moines les étudient

35. Brun CAndidus, Vita Aegil abbatis Fuldensis, lib. I, cap. 14-25 (éd. G. BeCht-JöRdens, p. 15-20).

36. J. E. RaAjmakers, Sacred Time, Sacred Space..., p. 149-150.

37. Ibid., p. 122.

38. Ibid., p. 129-131. 
toutes sans exception. Gereon Becht-Jördens a d'ailleurs montré que ce Codex regularum était connu et étudié à Fulda au début du $\mathrm{IX}^{\mathrm{e}}$ siècle $^{39}$.

Le second livre de la Vie d'Eigil est d'abord composé en vers asclépiades, puis, pour sa plus grande partie, en hexamètres ${ }^{40}$. S'il est vrai qu'il relate une seconde fois la vie d'Eigil, on y trouve tout de même des différences significatives: Brun ne rapporte plus les discussions des frères lors de l'élection du successeur de Ratgar, mais il évoque leurs opinions divergentes sous forme de figures allégoriques. La discordia est représentée comme une force hostile, à laquelle seule la prière commune des frères peut mettre un terme. Dans cette partie de l'œuvre, Brun ne s'intéresse pas aux faits, mais à leur signification, soit aux signa. En appliquant les préceptes de l'exégèse du De doctrina christiana de saint Augustin, il opte, dans la Vie métrique, pour la locutio figurata; il passe donc $\mathrm{du}$ «sens historique» au «sens mystique ${ }^{41}$. L'un des meilleurs exemples de l'usage de la locutio figurata se trouve dans la présentation de l'ancien abbé Ratgar comme une licorne au livre II, 5 ; ce passage a d'ailleurs été illustré d'une enluminure. Référence est ainsi faite au vers 22 du psaume 21 («sauvemoi des cornes des licornes ») : le père abbé qui avait divisé la communauté est représenté sous les traits de l'animal pour avoir effrayé son troupeau (les moines de Fulda). On peut même aller plus loin dans l'interprétation comme l'a proposé Gereon Becht-Jördens. Dans son commentaire des psaumes, Cassiodore identifie la licorne du psaume 21 à un persécuteur du Christ. Dès lors que l'abbé occupe, selon la Règle de saint Benoît, le rôle de «vicaire du Christ » ${ }^{42}$, on peut lire dans cette comparaison la critique la plus sévère possible de l'action de Ratgar. La solution à la crise vint de l'empereur qui donna à la communauté la permission d'élire un nouvel abbé. C'est ce que représente la troisième enluminure où l'on voit la légation, de retour de la cour impériale, chargée par Louis d'organiser une élection. L'image d'Eigil qui se dégage de cette deuxième partie de la Vie est celle d'un bon pasteur et d'un sapiens architectus du temple de Dieu ${ }^{43}$. L'illustration la plus claire de cette métaphore est fournie par le passage consacré à l'inauguration de la nouvelle basilique Saint-Sauveur en 819. Y participèrent non seulement tous les moines, mais aussi l'archevêque de Mayence, des évêques, des abbés et

39. Concernant les raisons qui ont mené à la division interne de la communauté de Fulda, voir G. Becht-Jördens, «Die Vita Aigil des Brun... », p. 41-45; J. E. RaAjumakers, Sacred Time, Sacred Space..., p. 57-61 et 101-103; M. GaILlaRd, D'une réforme à l'autre..., p. 158.

40. W. Berschin, Biographie und Epochenstil..., p. 247 et sq.

41. G. BeCht-JöRdens, Die Vita Aigil abbatis..., p. 42.

42. Ibid., p. 42-44; J. E. RaAiJmakers, Sacred Time, Sacred Space ..., p. 126-129: voir aussi Cassiodore, Expositio psalmorum, cap. 21-22 (éd. M. Adriaen, Turnhout, 1958 [CCSL, 97-98], p. 203- 532) ; et Benôit de Nursie, Regula Benedicti, cap. 2 (éd. A. De VogüÉ et J. NeufVILle, Paris, 1972 [Sources chrétiennes, 181], p. 440-442).

43. G. Becht-JöRdens, Die Vita Aegil abbatis..., p. 48. 
des comtes pour ne former qu' «un corps» in unum ${ }^{44}$. La consécration de l'église est ainsi présentée comme l'élévation d'un «temple de Dieu fait de pierres vivantes ${ }^{45} \gg$. Le peuple vit dans les bâtiments, mais Dieu vit dans les fidèles, et grâce à Eigil, le Saint-Esprit est entré dans un monument jusque-là symbole de discorde. La véritable Église est la communauté des moines, unie dans le Christ et réunie dans l'église ${ }^{46}$. Eigil est celui qui a bâti la véritable Église dans l'église en pierres, et pour donner toute la mesure de cette transformation, Brun n'hésite pas à parler d'un miracle ${ }^{47}$.

\section{La paix dans le cloître, la concorde dans l'Empire}

Destinées à la communauté de Fulda, les deux Vies d'Eigil le représentent comme un abbé idéal qui a dirigé sa communauté avec amour et sagesse, qui était à l'écoute de ses moines et qui a répondu aux exigences réformatrices de Louis le Pieux. Il a assumé le statut d'abbé d'un monastère royal - lieu de prière, de représentation et de soutien du pouvoir séculier -, tout en puisant l'inspiration de ses actions dans la Règle de saint Benoît ${ }^{48}$. Ces qualités sont mises en lumière par Brun à travers la crise qu'a représentée l'abbatiat de Ratgar, destitué par Louis le Pieux en 817 et remplacé par Eigil. En somme, la Vita prosaica développe l'idéal de vie d'un abbé à travers des discours et une exhortation prononcée par l'empereur. La Vita metrica favorise un discours ecclésiologique: en incarnant les vertus chrétiennes comme l'amour et la charité, Eigil serait arrivé à ériger la véritable Église de Dieu, celle qui est composée des fidèles.

44. Brun Candidus, Vita Aegil abbatis Fuldensis, lib. II, cap. 16: «Ecce repente / non mora digreditur propria de sede sacerdos / primum progrediens metropolitanus et exin / turba sacerdotum, abbatum comitumque potentum / ordinis atque etiam gaudens hinc inde minoris / ad tam magnificum et sanctum sub tempore festum / dispositio cum vi multa confluxit in unum » (éd. G. BECHT-JöRDENS, p. $55,1.560-566)$.

45. G. BeCht-JöRdens, Die Vita Aegil abbatis..., p. 39.

46. Il s'agit ici d'une pensée que Brun Candidus avait déjà exprimée dans le premier livre; Vita Aegil abbatis Fuldensis, lib. I, cap. 17: «Cuius tecturae princeps et conditor est Christus Iesus, fundamentum scilicet columnaque manens semper inmobilis perpetuae maiestatis virtute, in quo omnis aedificatio constructa crescit in templum sanctum in Domno (éd. G. BECHT-JöRDENS, p. 19).

47. Vita Aegil abbatis Fuldensis, lib. II, cap. 17: «Elevat interea populari voce repente / Advena plebs "Kyrie eleison", fit clamor ad astra / christicolis, ardent penitus fervore recenti / numinis infusi. Verum - mirabile dictu ! - / tu scis, non solito laudis vox omnibus aequa / efficitur: lacrymas profundunt gaudia mentis, / laetitiae, lacrymae et cantus miscentur in unum» (éd. G. BECHT-JöRDENS, p. 114-120); cf. J. E. RaAijmakers, Sacred Time, Sacred Space..., p. 129-131.

48. Comme fondateur de Fulda et modèle pour la communauté, Boniface se voit accorder un rôle moins important dans la Vie d'Eigil que dans celle de Sturmi: cf. J. E. RaAiJMakers, Sacred Time, Sacred Space..., p. 156. 
Ce faisant, Brun Candidus a beaucoup exagéré le rôle néfaste joué par Ratgar. Pour le présenter comme un anti-Eigil, incarnant la discordia, il a considérablement noirci son image. En fait, Louis le Pieux rappela Ratgar assez vite de son exil et l'ancien abbé de Fulda fut installé à la tête de la communauté de Frauenberg, à quinze minutes de marche de Fulda, où il vécut jusqu'à sa mort en 835. Cela n'aurait pas été possible si les relations entre l'ancien abbé de Fulda et sa communauté avaient été aussi mauvaises que Brun veut le faire croire ${ }^{49}$. En exagérant la mauvaise réputation de Ratgar, l'auteur poursuivait un but didactique et voulait donner à sa Vita Eigilis une forte valeur morale. Décrit comme celui qui apporte la paix et la concorde, Eigil devait servir de modèle absolu pour les moines. L'œuvre peut ainsi être qualifiée avec raison de «miroir des moines $»^{50}$. Dans le cas où leur comportement ne correspondait pas à l'idéal ici évoqué, ces derniers étaient appelés à se corriger. La Vie en prose était accessible à tous et il est fort probable qu'elle était lue à haute voix lors des offices ou des repas ; la Vie en vers s'adressait sans doute seulement aux frères les plus érudits du monastère. Il faut croire que Brun Candidus l'a écrite pour des esprits plus brillants, capables de lire et de comprendre un latin très recherché et de méditer, seuls, le sensus allegoricus de l'œuvre ${ }^{51}$. La Vita Eigilis n'a pas, ou pratiquement pas, circulé en dehors de Fulda et, hormis celui de l'abbaye, aucun catalogue de bibliothèque médiévale n'en fait mention ${ }^{52}$.

Il faut donc reposer la question du rapport entre la crise et la réforme que la Vie d'Eigil entendait accompagner. À première vue, on pourrait croire que le texte a été écrit pour clore la querelle de 816/817: après la discorde qui avait divisé les frères de Fulda, il fallait recréer l'unité de la communauté en lui proposant un modèle à suivre. Mais pourquoi Brun composa-t-il ce texte si tard? Autrement dit, pourquoi a-t-il fallu attendre presque vingt-cinq ans pour que l'on se préoccupât à Fulda d'apporter une solution littéraire à la crise ? Janneke Raaijmakers a clairement montré qu'Eigil avait écrit la Vita Sturmi au début de son abbatiat à cette fin précise. Comme nous l'avons expliqué plus haut, il rappelait dans ce texte les traditions bonifaciennes, l'importance de la règle de saint Benoît et la nécessaire collaboration avec les Pippinides pour les moines de Fulda. La Vie de Sturmi cherchait à recréer l'unité à Fulda et à clore la crise ouverte par Ratgar ${ }^{53}$. Dans ces conditions, les motivations de Brun Candidus ne pouvaient être exactement du même ordre.
49. Ibid., p. 135 .
50. Ibid., p. 144.
51. Ibid., p. 125 et 143 .
52. Ibid., p. 143.
53. Ibid., p. 68. 
Pour trouver des éléments de réponse, il faut analyser le contexte général des années trente du IX $\mathrm{X}^{\mathrm{e}}$ siècle. À cette époque, l'empire de Louis le Pieux connaissait une crise profonde: la naissance de Charles le Chauve en 823 avait provoqué un nouveau plan de partage de l'Empire que les autres fils de Louis n'avaient pas accepté. Les tensions culminèrent avec la destitution de Louis en 833, suivie de sa ré-investiture dans la charge impériale peu de temps après, ce qui provoqua sa vengeance vis-à-vis de ceux qui l'avaient trahi ${ }^{54}$. Au concile de Thionville/Diedenhofen, en 835, beaucoup d'évêques furent destitués de leurs sièges, ce qui suscita en retour une réaction des grands ecclésiastiques. Les Fausses décrétales - composées par Paschase Radbert ou sous sa direction, comme nous le savons maintenant - le furent certainement en réaction aux décisions du concile, car elles visent particulièrement le fait que l'empereur était intervenu contre les évêques sans l'accord du pape ${ }^{55}$. Les dernières années de Louis le Pieux furent marquées par de multiples guerres entre l'empereur et ses fils. Dans ces moments difficiles, Raban Maur et sa communauté restèrent fidèles à Louis le Pieux et c'est à Fulda que furent emprisonnés deux évêques rebelles, Ebbon de Reims et Goswin d'Osnabrück, en 834/835 ${ }^{56}$.

À Fulda même, la situation avait aussi connu des troubles dans les années 830. Lors du synode de Mayence, en 829, Gottschalk, qui était moine à Fulda, avait déclenché deux querelles théologiques majeures : il mit non seulement en question la pratique de l'oblation des enfants, mais il développa aussi une nouvelle théorie sur la double prédestination. Désireux de quitter Fulda, il expliqua qu'il avait été tonsuré, en tant que puer oblatus, contre sa volonté, tandis que Raban défendait la position selon laquelle l'oblation des enfants était voulue par Dieu et définitive. Quant à la double prédestination, Gottschalk soutenait l'idée que les uns étaient prédestinés au salut éternel et les autres à la damnation, ce qui était en contradiction avec la doctrine officielle de l'Église ${ }^{57}$. Raban considérait les positions du moine rebelle comme hérétiques, et en effet, ces dernières mettaient

54. Pour une synthèse très concise du règne de Louis le Pieux, voir J. SemmLER, «Ludwig der Fromme (814-840)», dans H. Beumann éd., Kaisergestalten des Mittelalters, Munich, 1985, p. 28-49; et aussi E. BoshoF, «Einheitsidee und Teilungsprinzip in der Regierungszeit Ludwigs des Frommen», dans P. Goldman et R. Collins éd. Charlesmagne's Heir. New Perspectives on the Reign of Louis the Pious (814-840), Oxford, 1990, p. 161-189.

55. Cf. notamment K. ZeChiEL-Eckes, «Ein Blick in Pseudoisidors Werkstatt. Studien zum Entstehungsprozess der falschen Dekretalen», Francia. Mittelalter, 28-1 (2001), p. $37-90$ (p. 55 sq.).

56. J. E. RAAiJmakers, Sacred Time, Sacred Space..., p. 155.

57. S. Patzold, «Raban, Gottschalk...»; voir également K. Vielhaber, Gottschalk der Sachse, Bonn, 1956; G. Morin, «Gottschalk retrouvé», Revue bénédictine, 43 (1931), p. 303-312; F. BRunHölzL, Histoire de la littérature latine ..., p. 115-120 et 290; et enfin K. ZeCHIEL-ECKEs, «Ein Dummkopf und Plagiator? Hrabanus Maurus aus der Sicht des Diakons Florus von Lyon», dans Raban Maur et son temps..., p. 119-136 (p. 120-133). 
en question les fondements de l'Église carolingienne: l'élite intellectuelle de l'époque était en grande partie issue des oblats; quand les parents offraient leurs enfants aux monastères, ils léguaient souvent en même temps leur héritage aux communautés et cette pratique assurait des revenus importants aux grands monastères; enfin, si la théorie d'une double prédestination s'était imposée, les efforts pastoraux de l'Église carolingienne auraient été fondamentalement mis en cause ${ }^{58}$. Même sans témoignage direct venu de l'intérieur de la communauté de Fulda, nous avons toutes les raisons de penser que le monastère avait été sérieusement affecté et divisé par ces querelles ${ }^{59}$. Cela semble confirmé par les donations faites à Fulda : après 831, elles diminuent; vers 835, quand Raban fait venir des reliques de martyrs de Rome, elles reprennent; puis, vers 840 , après la mort de Louis le Pieux, elles diminuent à nouveau ${ }^{60}$. Pour remédier à ces problèmes et recréer l'unité de la communauté, Raban Maur prit des mesures diverses, insistant sur un message ecclésiologique clair: recréer une véritable ecclesia au sens spirituel du terme. En faisant venir, en grande quantité, des reliques de Rome, en faisant repeindre les murs des bâtiments avec des scènes des Évangiles et des Vies de saints et en composant des tituli (des poèmes brefs en l'honneur des saints, inscrits sur les murs, les autels et les reliquaires), l'abbé voulut rendre visible la place de Fulda dans l'histoire du Salut et son lien avec la Jérusalem céleste ${ }^{61}$. Si Brun Candidus écrivit dans ce contexte une Vie qui mettait en scène une division d'une communauté monastique et qui montrait, à travers un discours ecclésiologique, comment cette crise avait trouvé une issue heureuse, il faut sans doute y voir une allusion à l'actualité des années 830 . Comme Raban Maur, Brun se souciait des problèmes internes dont souffrait sa communauté, notamment des divisions liées aux thèses de Gottschalk et à la temporaire baisse des donations qui suscitèrent certainement des controverses animées dans la communauté. L'abbatiat d'Eigil était alors un modèle à étudier et à suivre ${ }^{62}$. Voilà assurément une causa scribendi de l'œuvre de Brun Candidus.

On peut enfin penser que la Vita Eigilis avait d'autres destinataires: l'empereur Louis le Pieux et ses fils. Selon le texte de la Vie, la crise provoquée par Ratgar fut surmontée grâce à Louis qui, d'une certaine manière, est présenté comme le véritable dirigeant du monastère. Avec Eigil, il forme donc un «tandem» narratif à la manœuvre pour juguler la crise. Rappelons à ce sujet les actions

58. Voir note précédente, ainsi que J. E. RaAiJMAKers, Sacred Time, Sacred Space..., p. 188189.

59. Ibid., p. 189.

60. Ibid., p. 189-191.

61. Ibid., p. 179 sq.; voir également EAD., «Word, Image and Relics: Rabanus Maurus and the Cult of Saints (820s-840s)», dans Raban Maur et son temps..., p. 389-405.

62. Voir supra où nous avons parlé des lecteurs possibles de la Vie d'Eigil. 
et les discours prêtés à Louis dans la Vita: avant l'élection d'Eigil, l'empereur envoya ses missi pour qu'ils «corrigent» ${ }^{63}$ les frères, puis il insista sur le fait que les frères devaient s'aimer mutuellement (en appliquant la regula individua caritatis $\left.{ }^{64}\right)$ et $«$ rester loin de toute forme de discorde ${ }^{65}$, car «Dieu n'est pas un Dieu de la dispute, mais de la paix ${ }^{66}$. Plus loin, Louis aurait rappelé que les moines devaient «servir Dieu et la concorde ${ }^{67}$. Il ne fait pas de doute qu'Eigil répondait parfaitement aux attentes de l'empereur: il était compréhensif, sévère et doux à la fois ${ }^{68}$; surtout, il prenait toutes les décisions après avoir consulté ses frères ${ }^{69}$, soucieux de créer un consensus permanent. Il est sûr que Brun et sans doute aussi Louis le Pieux - aurait aimé que les fils de l'empereur se comportent exactement comme l'abbé de Fulda. Avec plus de concordia et de caritas entre les héritiers du trône, il aurait été possible de préserver l'unité et la paix : c'est sans aucun doute ce message que Brun voulait faire passer. Les puissants de l'Empire - en premier lieu les fils de Louis le Pieux qui étaient en discorde permanente - auraient bien fait de s'inspirer de la manière dont les abbés dirigeaient leur communauté. Brun Candidus manifestait de la sorte une «conscience de la crise» dans laquelle l'Empire se trouvait alors. La solution qu'il proposait s'inspirait de la vie monastique ${ }^{70}$. N'oublions pas non plus que Brun Candidus n'a pas hésité à critiquer indirectement et prudemment Louis le Pieux dans la Vita Eigilis: il fait prononcer à l'empereur un discours sur l'abbé

63. Brun Candidus, Vita Aegil abbatis Fuldensis, lib. I, cap. 3: «[...] fraterna dilectione praemonendo corrigerent» (éd. G. BEChT-JördENs, p. 5); dans la Vie en vers (lib. II, cap. 6), on peut lire: «Misit ab occiduo hic monachos, qui maxime nostros? / quin et finitimos fratres hinque inde cubantes? / mores et studia et leges et dogmata patrum / incaepta incipere et lapsa renovare monerent» (éd. G. BeChT-JöRDENS, p. 40).

64. Brun CAndidus, Vita Aegil abbatis Fuldensis, lib. I, cap. 9: «Vacat enim dilectio Dei, nisi subjungatur et proximi. Plenum ergo praeceptum est veritatis, ut maneat in sensu individua regula caritatis» (éd. G. BECHT-JöRDENS, p. 9).

65. Ibid.: «Ipsi sunt, qui manducant panem in regno Dei, sine macula vivunt et alienae ruga discordiae».

66. Ibid.: «Dissentionis, si quas habetis inter vos, proiicite ab invicem scientes Deum non esse seditionis, sed pacis» (éd. G. BECHT-JöRDENS, p. 10).

67. Ibid. : « ...ut serviatis Deo et concordiae...»

68. Brun CAndidus, Vita Aegil abbatis Fuldensis, lib. I, cap. 6 (éd. G. BeCht-Jördens, p. 8), où l'on trouve un portait d'Eigil.

69. Brun CANDidus, Vita Aegil abbatis Fuldensis, lib. I, cap. 19: «Omnia fac cum consilio» (éd. G. BeChT-JöRdens, p. 17).

70. J. E. RAaiJMAKERs, Sacred Time, Sacred Space..., p. 158, est ici plus prudente. Elle mentionne les crises des années 830 , mais seulement comme arrière-fond, peut-être parce que déjà, à l'époque de Charlemagne, les capitulaires avaient exigé la concorde entre les fils: voir par exemple l'Admonitio generalis, cap. 62 (éd. A. BoRETius, Capitularia regum Francorum, MGH, Leges, Hanovre, 1883, p. 58). L'intensité avec laquelle la concorde est exigée dans la Vie d'Eigil mérite cependant d'être relevée. 
idéal qui doit être personnellement un modèle pour ses frères. Autrement dit, l'abbé idéal doit être moine. Ce sont des mots que l'empereur n'a bien entendu jamais prononcés, car il investissait régulièrement des abbés laïques de son choix. Brun suggère à l'empereur ce qu'il attendait de lui : n'investir que des moines à l'abbatiat. On peut alors imaginer qu'il souhaitait donner à Louis et à ses fils, à travers la Vita Eigilis, un conseil, indirect mais clair, pour qu'ils assurent, dans les monastères et dans l'Empire, un gouvernement agréable à Dieu.

Cette interprétation n'est, certes, pas prouvée car il nous manque des passages plus explicites, mais elle est d'autant plus probable que le discours de Brun Candidus correspond à d'autres textes plus directement en rapport avec la querelle entre l'empereur et des fils. Parmi eux, il y a des œuvres de Raban Maur, l'abbé de Brun, à l'approbation duquel la Vie d'Eigil avait été soumise. En effet, dans ses écrits comme le Liber de reverentia filiorum erga patris et subditorum et le Liber des virtutibus et vitiis, datées de 834, l'abbé de Fulda met en avant une idéologie qui construit la communauté politique autour de la caritas, de la fides et de la concordia. Raban proclame un idéal de paix et préconise une société de frères unis par un amour filial ${ }^{71}$. Mayke de Jong a récemment montré à quel point les termes de pax, concordia, fides, caritas, mais aussi admonitio et correctio étaient omniprésents dans les discours politiques de l'époque ${ }^{72}$. Par ailleurs, il n'est aucunement improbable qu'une Vie illustrée ait été envoyée à l'empereur, comme le montre l'exemple plus tardif de la Chronique universelle illustrée d'Otton de Freising adressée à l'empereur Frédéric Barberousse ${ }^{73}$. Envoyer un texte à valeur pédagogique, illustré de surcroît, à un souverain, n'était donc peutêtre pas une pratique si rare.

La Vie d'Eigil, qui traite de la crise survenue à Fulda à la suite des réformes monastiques de Benoît d'Aniane en 816, est donc bel et bien une Vie destinée à «réformer» ou plutôt à «corriger» les moines. Mais contrairement à ce qu'on pourrait penser, l'auteur ne vise pas tant à donner une solution aux réformes qu'il a présentées dans son œuvre, mais à des crises bien plus tardives qui éclatent dans les années 830 , liées aux controverses agitées par Gottschalk ainsi qu'à la diminution des donations. Peut-être Brun entendait-il aussi dénoncer la crise

71. Cf. G. BüHreR-ThierRy, «Raban Maur et l'épiscopat de son temps», dans Raban Maur et son temps..., p. 63-76 (p. 72-73).

72. M. De Jong, The Penitential State, Authority and Atonement in the Age of Louis the Pious (814-840), Cambridge, 2009, passim.

73. Le manuscrit Jena, ThULB, Ms Bos. q. 6, fut sans doute directement copié sur ce modèle: cf. F. NAGEL, «Otto von Freising, Chronica sive historia de duabus civitatibus. Das Jenaer Manuscript (Ms. Bos. q. 6) und seine Illustrationen », dans M. WALlRafF éd., Weltzeit, christliche Weltchronistik aus zwei Jahrtausenden in Beständen der Thüringer Universitäts- und Landesbibliothek Jena, Berlin/ New York, 2005, p. 32-58; G. BEChT-JöRDENs éd., Vita Aegil..., p. xxix, évoque d'ailleurs l'hypothèse selon laquelle un manuscrit de la Vita Eigilis aurait été destiné à Louis le Pieux. 
dans laquelle se trouvait l'Empire, déchiré par la guerre civile qui opposait Louis le Pieux et ses fils. À tous, l'hagiographe proposait un modèle de bonne gouvernance, fondée sur la concorde, la paix, l'amour, la charité, la sagesse, la pratique de la pénitence, la correction et la recherche du consensus.

Klaus KRönerT - Université Lille Nord de France UDL3 (IRHiS UMR 8529), BP 149, F-59653 Villeneuve d'Ascq Cedex

\section{Réformer la vie monastique ou réformer l'Empire? La Vie d'Eigil de Fulda par Brun Candidus (vers 840)}

Quelle fonction la Vie d'Eigil de Fulda (BHL 2440-2441), rédigée vers 840 par Brun Candidus, pouvait-elle avoir dans la société de l'époque ? Le texte relate de manière détaillée, en prose et en vers, avec de surcroît une série d'enluminures, la situation critique que la communauté de Fulda a connue lors des réformes de 816/817 sous l'abbatiat de Ratgar, la destitution de ce dernier par Louis le Pieux, l'élection du nouvel abbé, Eigil, ainsi que son abbatiat qui parvint à sortir la communauté de la crise. Eigil est décrit comme un abbé qui a dirigé ses moines avec amour, sagesse et fermeté, qui a été à l'écoute des frères et qui a parfaitement répondu aux exigences de l'empereur, de sorte qu'il fit régner la paix et la concorde. Ce n'est cependant pas la crise de 816/817 qui préoccupait Brun Candidus lors qu'il écrivit cette Vie, mais les crises dans lesquelles la communauté de Fulda et l'Empire se trouvaient plongés dans les années 830: les conflits liés aux thèses hérétiques du moine Gottschalk et la guerre civile entre Louis le Pieux et ses fils. À tous les acteurs de cette nouvelle période sombre, l'hagiographe proposait un modèle de bonne gouvernance, fondée sur la concorde, la charité, la sagesse, mais aussi sur la pratique de la correction et la recherche du consensus.

Fulda - abbé - monastère - réforme - Brun Candidus.

\section{Reforming Monastic Life or Reforming Empire? The Life of Eigil of Fulda by Brun Candidus (c. 840)}

The Life of Eigil of Fulda (BHL 2440-2441), written by Brun Candidus (about 840 ), is focused on the function that this Life devoted to the fourth abbot of Fulda had in the society of that time. The text relates with many details, in prose and in verses as well as a series of miniatures, the critical situation which the community of Fulda had experienced during the reform of 816/817 under abbot Ratger, his deposition by Louis the Pious, the election of a new abbot, Eigil, and how the latter succeeded in guiding his community out of the crisis. Eigil 
is described as an abbot who was leading his monks with love, wisdom and firmness, who was listening to his brothers and who came up the expectations of the emperor, so that he restored peace and concord. However, it was not the crisis of $816 / 817$ that was worrying Brun Candidus, when he was writing this Life, but the new crises in which the community of Fulda and the Empire were involved in the third decade of the 9th century: i.e. the conflict concerning the heretic positions of the monk Gottschalk and the civil war among Louis the Pious, his sons and their allies. The hagiographer proposed to all these actors of this new dark period a model of a good government, founded on concord, charity, wisdom, the practice of correction and the quest for consensus.

Fulda - abbot - Brun Candidus - hagiography - monastery - reform. 
